\title{
Thermal leptogenesis in extended supersymmetric seesaw model
}

\author{
M. Hirsch* and J. W.F. Valle \\ Instituto de Física Corpuscular-C.S.I.C./Universitat de València Campus de Paterna, Apt 22085, E-46071 València, Spain \\ M. Malinský ${ }^{*}$ \\ School of Physics and Astronomy, University of Southampton, SO16 1BJ Southampton, United Kingdom \\ J. C. Romão ${ }^{\S}$ \\ Departamento de Física and CFTP, Instituto Superior Técnico Av. Rovisco Pais 1, 1049-001 Lisboa, Portugal \\ U. Sarkar"l \\ Physical Research Laboratory, Ahmedabad 380 009, India \\ (Received 10 August 2006; published 29 January 2007)
}

\begin{abstract}
We consider an extended supersymmetric SO(10) seesaw model with only doublet Higgs scalars, in which neutrino masses are suppressed by the scale of $D$-parity violation. Leptogenesis can occur at the $\mathrm{TeV}$ scale through the decay of a singlet $\Sigma$, thereby avoiding the gravitino crisis. Washout of the asymmetry can be effectively suppressed by the absence of direct couplings of $\Sigma$ to leptons.
\end{abstract}

DOI: 10.1103/PhysRevD.75.011701

One of the most attractive scenarios to account for the baryon-to-photon ratio of the universe is leptogenesis [1,2] in the context of the seesaw mechanism [3-7]. According to this the out-of-equilibrium decays of the lightest righthanded (s)neutrinos produce a net lepton number which is later reprocessed into the observed baryon asymmetry. However, if they are thermally produced in the early universe, the reheating temperature $\left(T_{R}\right)$ should exceed $\sim 10^{9} \mathrm{GeV}[8,9]$. In the context of supersymmetry, this leads to the overproduction of gravitinos, with catastrophic consequences for the evolution of the universe [10]. Although somewhat model-dependent, the upper bound on $T_{R}$ from gravitino overproduction is rather stringent and can be as strong as $T_{R}<10^{6-7} \mathrm{GeV}$ [11].

Here we suggest a way to cure this problem by extending the seesaw mechanism. In contrast to Ref. [12] we keep $R$-parity conserved and adopt a supersymmetric grand unified $\mathrm{SO}(10)$ model, already proposed in [13]. The model requires three sequential gauge singlet superfields $S_{i}, i=$ $1,2,3$ in addition to the three usual fermions in the 16dimensional representation of $\mathrm{SO}(10)$ [14]. In contrast to conventional seesaw, the left-right symmetry is broken only by Higgs doublets [15-17]. One remarkable feature of these models is that the scale of neutrino masses is independent of the $(B-L)$ breaking scale [13]. We assume an additional singlet superfield $\Sigma$ without direct couplings to the usual matter multiplets. It is the out-ofequilibrium decay of this superfield $\Sigma$ that drives lepto-

\footnotetext{
*Electronic address: mahirsch@ific.uv.es

†Electronic address: valle@ific.uv.es

URL: http://ahep.uv.es

${ }^{\ddagger}$ Electronic address: malinsky@ phys.soton.ac.uk

${ }^{\S}$ Electronic address: jorge.romao@ist.utl.pt

"Electronic address: utpal@prl.res.in
}

PACS numbers: 12.10.Dm, 12.60.Jv, 14.60.St, 98.80.Cq

genesis. Its mass can be as low as $\mathrm{TeV}$, thus avoiding conflict with reheating bounds [11]. Moreover, in contrast to the simplest, unextended seesaw, one can naturally suppress erasure of the created asymmetry due to washout processes without conflicting with the magnitude of neutrino masses indicated by oscillation experiments [18].

The present model spontaneously violates $D$-parity, which connects the subgroups $S U(2)_{L}$ and $S U(2)_{R}$ of $\mathrm{SO}(10)$. In the left-right symmetric models the $D$-parity is identified with the usual parity operation of the Lorentz group. Thus the vacuum expectation value (vev) of any $D$-parity odd singlet scalar can break parity spontaneously even when $S U(2)_{R}$ is unbroken. This can have important implications, for example, it makes the $S U(2)_{L}$ and $S U(2)_{R}$ gauge couplings differ, and similarly the masses of lefthanded and the corresponding right-handed fields. In an $\mathrm{SO}(10)$ grand unified theory, the adjoint and the 210 representations contain $D$-parity odd singlet fields that can break parity spontaneously [19].

Here we consider the symmetry breaking pattern

$$
\begin{aligned}
S O(10) & \stackrel{45210}{\rightarrow} S U(3)_{c} \times S U(2)_{L} \times S U(2)_{R} \times U(1)_{B-L} \\
& \stackrel{\chi_{R}}{\rightarrow} S U(3)_{c} \times S U(2)_{L} \times U(1)_{Y} \\
& \stackrel{\chi_{L}, \phi}{\rightarrow} S U(3)_{c} \times U(1)_{Q} .
\end{aligned}
$$

We also impose a global $U(1)_{G}$ symmetry, under which all three minimal $\mathrm{SO}(10)$ matter supermultiplets (superfields with odd matter parity) in the $\mathbf{1 6}$ are neutral and the gauge singlet matter superfields $S_{i}, i=1,2,3$ carry nonzero $G$-charge. In addition, we introduce singlet chiral superfields $\Sigma$ and $X$, the latter with $G=0$ and invariant under $D$-parity.

For the symmetry breaking we consider the minimum number of Higgs scalars. In addition to the adjoint, we 
break the group $\mathrm{SO}(10)$ with a 210-representation, which also contains a $D$-parity odd singlet $\sigma \equiv(1,1,1,0) \subset \mathbf{2 1 0}$. The left-right symmetry is broken by a 16-plet of Higgs (this contains $\chi_{R}$ and $\chi_{L}$ ) with $G$-charge opposite to that of the singlet matter fields $S_{i}$. The electroweak symmetry is broken by a 10-plet $(\phi)$ of $\mathrm{SO}(10)$, neutral under $G$, which contains the usual bi-doublet field. Under the left-right symmetric subgroup $G_{L R} \subset \mathrm{SO}(10)$ the transformations of the remaining fields responsible for symmetry breaking are $\phi \equiv(1,2,2,0) \subset \mathbf{1 0}, \chi_{R} \equiv(1,1,2,1) \subset \mathbf{1 6}$ and $\chi_{L} \equiv$ $(1,2,1,1) \subset \mathbf{1 6}$. The electric charge assignment and $U(1)$ normalization are,

$$
Q=T_{3 L}+T_{3 R}+\frac{B-L}{2}=T_{3 L}+\frac{Y}{2} .
$$

The Yukawa couplings relevant for neutrino masses are

$$
\begin{aligned}
\mathcal{L}_{Y}= & Y_{i j} N_{i L} \nu_{j L} \phi+F_{i j} \nu_{i L} S_{j} \chi_{L}+\tilde{F}_{i j} N_{i L} S_{j} \chi_{R} \\
& +F_{X} X . \Sigma \Sigma,
\end{aligned}
$$

Note that a direct Majorana mass term for the singlet fields $S_{i}$ is forbidden by the $U(1)_{G}$ quantum number and the fact that the only singlet scalar $\sigma$ is odd under $D$-parity, while $S_{i} S_{j}$ are even under $D$-parity. For the same reason, $\sigma$ cannot couple to $\Sigma$, although the vev of the scalar in $X$ gives a bare mass for $\Sigma, M_{\Sigma}=f_{X}\langle X\rangle$, of the order of TeV. In contrast to the singlet superfield $\Sigma, X$ is even under matter parity and hence can have couplings like $X \chi_{L}^{c} \chi$, where $\chi_{L}^{c}$ is a left-chiral superfield transforming as $(1,2,1,-1)$ that is required for anomaly cancellation. Then the quartic couplings of the scalar components of these fields $\tilde{X}^{\dagger} \tilde{X} \tilde{\chi}_{L}^{\dagger} \tilde{\chi}_{L}$ will produce the field $X$ thermally, which in turn can produce the singlet fields $\Sigma$ at $T>M_{\Sigma}$. We also introduce a soft term breaking $U(1)_{G}$, which allows the term $\Sigma S_{i}$ mixing the scalar components of these fields.

This will then give a $10 \times 10$ neutrino mass matrix, in the basis $\left(\nu_{i}, \Sigma, N_{i}, S_{i}\right)$ :

$$
M_{\nu}=\left(\begin{array}{cccc}
0 & 0 & Y v & F v_{L} \\
0 & M_{\Sigma} & 0 & \Delta^{T} \\
Y^{T} v & 0 & 0 & \tilde{F} v_{R} \\
F^{T} v_{L} & \Delta & \tilde{F}^{T} v_{R} & 0
\end{array}\right)
$$

where, $v=\langle\phi\rangle, v_{L}=\left\langle\chi_{L}\right\rangle$ and $v_{R}=\left\langle\chi_{R}\right\rangle$ are the vevs for the fields $\phi, \chi_{L}$ and $\chi_{R}$ respectively and $\Delta$ is the $U(1)_{G}$ breaking entry. This mass matrix will give two heavy states which are dominantly the right-handed neutrino $N_{i L}$ and the singlets $S_{i}$, with a lighter state $\Sigma$. Using the seesaw diagonalization prescription given in Ref. [6] we obtain the effective left-handed light neutrino mass matrix as

$$
m_{\nu}=\frac{1}{M_{\Sigma}} G G^{T}-\left[Y\left(F \tilde{F}^{-1}\right)^{T}+\left(F \tilde{F}^{-1}\right) Y^{T}\right] \frac{v v_{L}}{v_{R}}
$$

where $G \equiv Y\left(\tilde{F}^{-1}\right)^{T} \frac{v \Delta}{v_{R}}$.

The first contribution in Eq. (4) arises from the soft $U(1)_{G}$ breaking term. In order to keep the absolute neutrino mass scale in the $\mathrm{eV}$ range one should require

$$
\frac{v^{2}|\Delta|^{2}}{M_{\Sigma} v_{R}^{2}} \lesssim \mathrm{eV},
$$

indicating the need for the smallness of $G$-violation.

We now turn to the second term. First note that its structure is different from the conventional seesaw, first that it is linear in the Yukawa coupling $Y$ [13]. In order to discuss its magnitude we consider the minimization of the most general scalar potential. This will determine the vevs of the different fields:

$$
\langle\phi\rangle=v ; \quad\left\langle\chi_{L}\right\rangle=v_{L} ; \quad\left\langle\chi_{R}\right\rangle=v_{R} ; \quad\langle\sigma\rangle=\eta .
$$

In models of $D$-parity violation it is usual to choose the parameters of the potential to make the masses of the lefthanded and right-handed fields different. A similar prescription also holds in the presence of superymmetry, so that we can have $D$-parity violation at a high scale, whereas the $B-L$ symmetry is broken at a scale that can be as low as the electroweak symmetry breaking scale. Since the $D$-parity breaking scale is much higher than the scale at which the left-right symmetry breaks, and this in turn is higher than the electroweak symmetry breaking scale, one has the "vev-seesaw" relation

$$
v_{L} \propto \frac{v_{R} v}{M_{U}},
$$

where $M_{U}$ is determined by the $\mathrm{SO}(10)$ breaking vevs, so that the second contribution to the neutrino mass in Eq. (4) becomes naturally small, suppressed by the unification scale, irrespective of the $(B-L)$ violating scale $v_{R}$ which can be rather low [13]. This is in sharp contrast to the conventional left-right symmetric seesaw models.

Note that in the present model we have a $U(1)_{G}$ global symmetry, which is broken by the vev $\left\langle\chi_{R}\right\rangle$ and also explicitly through the soft $\Sigma S$ bilinear mixing terms. The corresponding would-be Goldstone picks up a large mass and/or can be made invisible in case all $G$-breaking comes spontaneously.

All in all, one can have naturally small neutrino masses independent of the magnitude of the $(B-L)$ symmetry breaking scale, which may be as low as the TeV scale.

We now discuss the issue of leptogenesis in this model. It can occur only after the local $(B-L) \subset \mathrm{SO}(10)$ symmetry is broken. It will take place through the decay of the singlet fermion $\Sigma$. In order to get the total width of $\Sigma$ decaying to a lepton-Higgs pair via the mixing with the $N$ and $S$ fields one should transform the relevant superpotential term from the defining basis $(\nu, \Sigma, N, S)$ to the physical matter and Higgs doublet fields and identify the effective Yukawa coupling of $\Sigma$ to the light lepton-Higgs pair $L H$. This way the total width of $\Sigma$ is given by (treating $Y_{\Sigma}$ as a column vector)

$$
\Gamma_{\Sigma} \sim \frac{1}{8 \pi} Y_{\Sigma}^{\dagger} Y_{\Sigma} M_{\Sigma}
$$


THERMAL LEPTOGENESIS IN EXTENDED ...

In order to estimate $Y_{\Sigma}$ we need to compute the projection $U_{\nu^{c} \Sigma}$ of $\Sigma$ onto the $N$ 's. This will determine the relevant effective coupling of the $\Sigma$ to $L H$ pair. The $\mathcal{O}(1)$ coefficient $\alpha_{H}$ denotes the projection of the relevant light minimal supersymmetric standard model (MSSM) Higgs doublet $h$ into the directions of the defining (up-type) Higgs doublets living in $H \in 10_{H}$. We leave it unspecified as the full-featured analysis of the Higgs potential is out of the scope of this work.

In order to do that let us use again the perturbative seesaw diagonalization prescription of Ref. [6]. The method is especially convenient to the discussion of leptogenesis, as it includes all $C P$ phases. One finds, at leading order:

$$
U_{N \Sigma}=\left(\tilde{F}^{-1}\right)^{T} \frac{\Delta}{v_{R}}, \quad Y_{\Sigma}=\alpha_{H} Y U_{N \Sigma}
$$

Note that the $G$-breaking quantity $\Delta$ determines $U_{N \Sigma}$ and $Y_{\Sigma}$. This can be easily understood from the Feynman diagrams for the effective Yukawa coupling $Y_{\Sigma}$, cf. Fig. 1. The figure illustrates the (lowest order) treelevel graph giving rise to the effective $\Sigma L H$ Yukawa interaction. Notice that this decay can occur out-ofequilibrium for moderately large values of the Yukawa couplings since, for sufficiently small values of the parameter $\Delta$, the effective $L H N$ vertex will be suppressed, as seen from the graph.

The decay width of $\Sigma$ is estimated as

$$
\Gamma_{\Sigma}=\frac{\alpha_{H}^{2}}{8 \pi} \frac{\Delta^{\dagger}}{v_{R}}\left(\tilde{F}^{-1}\right)^{\dagger} Y^{\dagger} Y\left(\tilde{F}^{-1}\right) \frac{\Delta}{v_{R}} M_{\Sigma}
$$

The interference of one loop diagrams and tree level diagrams (see Fig. 2) generates a lepton asymmetry [20]. Keeping only the contribution of the lightest $N_{1}^{ \pm}$pair we obtain the following estimate for the $C P$ asymmetry produced in the decay of $\Sigma$,

$$
\varepsilon_{\Sigma} \propto-\frac{3}{16 \pi} \frac{M_{\Sigma}}{M_{1}} \frac{\operatorname{Im}\left[\left(Y_{\Sigma}^{\dagger} F_{k} U_{S} \alpha_{\chi^{k}}\right)_{1}\left(Y_{\Sigma}^{\dagger} Y U_{N} \alpha_{H}\right)_{1}\right]}{Y_{\Sigma}^{\dagger} Y_{\Sigma}},
$$

where $\alpha_{\chi^{k}}$ are the projections of the light MSSM-like

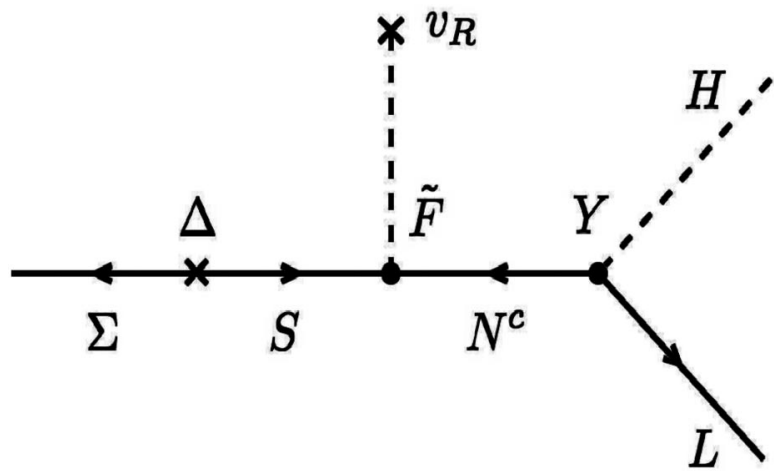

FIG. 1. Lowest order tree-level graphs giving rise to the effective $\Sigma L H$ Yukawa interaction; for more details see text.
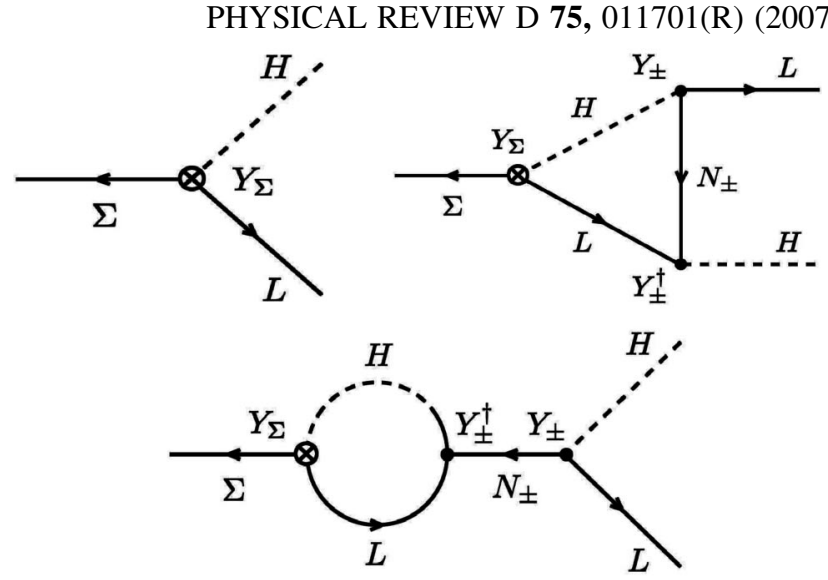

FIG. 2. Tree level and one loop diagrams for the decay of $\Sigma$ that interferes to generate a lepton asymmetry of the universe.

Higgs doublet onto the defining Higgs doublets in the $\overline{16}_{H}^{k}$ and $M_{1}$ is the mass of the (almost degenerate) lightest pair of the $N_{ \pm} \equiv \frac{1}{\sqrt{2}}(N \pm S)$ states. In addition

$$
U_{N}^{T} \tilde{F} v_{R} U_{S}=\operatorname{diag}\left(M_{1}, M_{2}, M_{3}\right),
$$

The main feature of this scenario is that the suppression factor of $\Delta / v_{R}$ does not enter in the amount of asymmetry generated in the decays of $\Sigma$. At the time of decays of $\Sigma$, the number densities of the right-handed neutrinos $N_{i}$ and the singlets $S_{i}$ should be fairly less so that they do not wash out the asymmetry generated by the decay of $\Sigma$, which is subsequently converted to a baryon asymmetry by the sphaleron processes. Since the neutrino masses are maintained small by the scale of $D$-parity violation, and thermal production of $\Sigma$ depends only on its coupling to $X, F_{X}$, there is no restriction from neutrino masses on the couplings $Y_{\Sigma}$, which are dependent on the scale of $B-L$ violation. Couplings of the chiral superfield $X$ with other fields including $\phi$ produce them thermally, while $\Sigma$ has no other couplings.

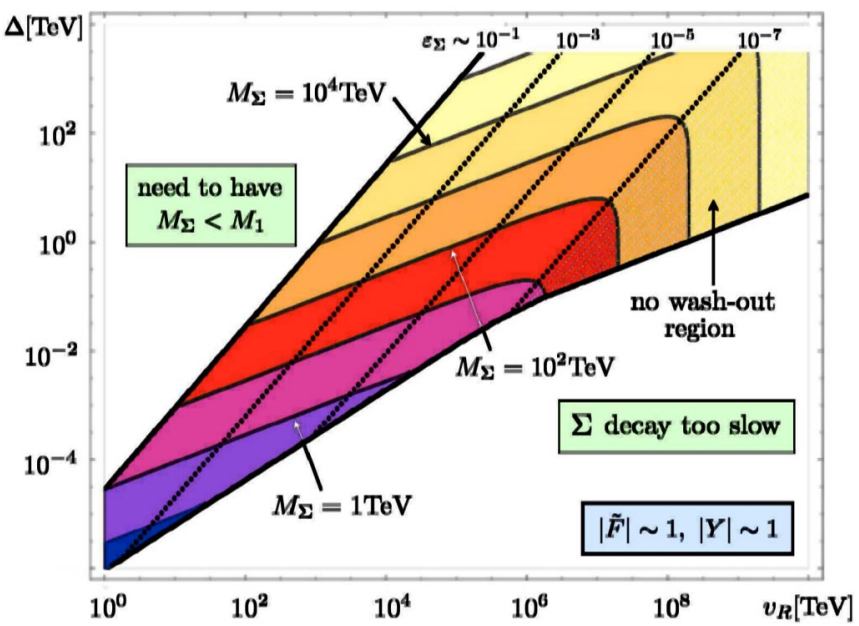

FIG. 3 (color online). Leptogenesis parameter region (see text). 
In order to induce successful leptogenesis the $\Sigma$ must decay before the electroweak phase transition. Moreover $\Sigma$ decay must take place out-of-equilibrium, i.e. one must fulfill the condition $\Gamma_{\text {Hubble }}>\Gamma_{\Sigma}>\Gamma_{\text {sphaleron. }}$.

Figure 3 shows the typical correlations among the magnitudes of parameters $v_{R}, \Delta$ and $M_{\Sigma}$ leading to roughly $\eta \sim \varepsilon_{\Sigma} \kappa / g_{*} \sim 6 \times 10^{-10}$, where $g_{*}$ is the relevant number of degrees of freedom $\sim 2 \times 10^{2}, \kappa$ is 1 if the width is well below the Hubble rate, and falls exponentially otherwise.

Note that the lepton asymmetry $Y_{L}=\epsilon \kappa / g_{*}$ is defined as $\left(n_{L}-n_{\bar{L}}\right) / s$ with $s$ being the entropy. The conversion of $L$ into $B$ brings another factor $1 / 3$ or so. Next, we expect the efficiency factor $\kappa$ to decay exponentially with the amount of out-of-equilibrium condition (OEC) violation. More quantitatively, for $\Gamma_{\Sigma} \sim$ $\left.10^{x} \sqrt{4 \pi g_{*} / 45}\left(T^{2} / M_{P l}\right)\right|_{T=M_{\Sigma}}$ the OEC is satisfied as long as $x \lesssim 0$ and we assume the efficiency factor to behave like $\kappa(x) \sim\left(1+10^{x+1}\right)^{-1}$ corresponding to essentially no washout for $\Gamma_{\Sigma}$ well below the Hubble expansion rate while decaying exponentially with the amount of the OEC violation for $x \geq 0$, cf. [21].

In our estimates we assume all Yukawas are order unity, e.g. $|\tilde{F}| \sim|Y| \sim 1$. For a given $v_{R}$ there is only a certain range for $M_{\Sigma}$ : (i) the lower bound indicated by the lower solid line comes from the need to generate enough asymmetry (proportional to $M_{\Sigma} /|\tilde{F}| v_{R}$ ) while (ii) the upper bound stems from the need to have $M_{\Sigma}$ below $M_{1}$. Note that in the upper right region washout is negligible, here the asymmetry $\varepsilon_{\Sigma}$ is essentially constant as $\sim M_{\Sigma} /|\tilde{F}| v_{R}$. On the other hand, in the region left of the dotted line with $\varepsilon_{\Sigma}=10^{-7}$ one would have too large an asymmetry, $\varepsilon_{\Sigma}>$ $10^{-7}$, were it not for the fact that, in this region, this is compensated by a certain amount of washout, so as to lead to an acceptable asymmetry. Note also the relative smallness of the $G$-breaking $\Delta$ parameter. One sees, for example, that successful leptogenesis can occur for $M_{\Sigma}=1 \mathrm{TeV}$ and low $v_{R}=10 \mathrm{TeV}$.

In short, we have considered a supersymmetric $\mathrm{SO}(10)$ seesaw model with only doublet Higgs scalars, in which neutrino masses are suppressed by the scale of $D$-parity violation, regardless of the value of the $(B-L)$ violating scale, which can be low. This would allow for the existence of new physics (e.g. a $Z^{\prime}$ gauge boson) accessible at accelerators. Leptogenesis can occur at the $\mathrm{TeV}$ scale through the decay of a singlet $\Sigma$, thereby avoiding the gravitino crisis. Washout of the asymmetry is suppressed by the absence of direct couplings of $\Sigma$ to leptons.

Note that the mechanism described here involving the addition of the $\Sigma$ field is very natural in the framework of the extended seesaw model but not in the simplest type-I seesaw scheme [22]. Details of the mechanism and a critical comparison with unextended seesaw schemes will be presented elsewhere [23].

Work supported by MEC Grants No. FPA2005-01269 and BFM2002-00345, by EC Contracts RTN network MRTN-CT-2004-503369 and ILIAS/N6 RII3-CT-2004506222. M. H. is supported by a Ramon y Cajal grant.
[1] M. Fukugita and T. Yanagida, Phys. Lett. 174B, 45 (1986).

[2] W. Buchmuller, R. D. Peccei, and T. Yanagida, Annu. Rev. Nucl. Part. Sci. 55, 311 (2005).

[3] P. Minkowski, Phys. Lett. 67B, 421 (1977).

[4] Articles by M. Gell-Mann, P. Ramond, R. Slansky, T. Yanagida, R. Mohapatra, and G. Senjanovic, in Proc. of Int. Conf. on the Seesaw Mechanism and the Neutrino Mass, Paris, France, 2004, edited by J. Orloff, S. Lavignac, and M. Cribier.

[5] J. Schechter and J.W.F. Valle, Phys. Rev. D 22, 2227 (1980).

[6] J. Schechter and J.W.F. Valle, Phys. Rev. D 25, 774 (1982).

[7] G. Lazarides, Q. Shafi, and C. Wetterich, Nucl. Phys. B181, 287 (1981).

[8] W. Buchmuller, P. Di Bari, and M. Plumacher, Ann. Phys. (N.Y.) 315, 305 (2005).

[9] G. F. Giudice et al., Nucl. Phys. B685, 89 (2004).

[10] M. Y. Khlopov and A. D. Linde, Phys. Lett. 138B, 265 (1984).

[11] M. Kawasaki, K. Kohri, and T. Moroi, Phys. Rev. D 71, 083502 (2005).

[12] Y. Farzan and J. W. F. Valle, Phys. Rev. Lett. 96, 011601 (2006).
[13] M. Malinsky, J. C. Romao, and J. W. F. Valle, Phys. Rev. Lett. 95, 161801 (2005).

[14] R. N. Mohapatra and J. W. F. Valle, Phys. Rev. D 34, 1642 (1986).

[15] E. Akhmedov, M. Lindner, E. Schnapka, and J. W. F. Valle, Phys. Rev. D 53, 2752 (1996).

[16] S. M. Barr and I. Dorsner, Phys. Lett. B 632, 527 (2006).

[17] T. Fukuyama, A. Ilakovac, T. Kikuchi, and K. Matsuda, J. High Energy Phys. 06 (2005) 016.

[18] M. Maltoni, T. Schwetz, M. A. Tortola, and J. W. F. Valle, New J. Phys. 6, 122 (2004); Appendix C in hep-ph/ 0405172 (v5) provides updated results which take into account all developments as of June 2006, namely: new SSM, new SNO salt data, latest K2K and MINOS data; previous works by other groups are referenced therein.

[19] D. Chang, R. N. Mohapatra, and M. K. Parida, Phys. Rev. Lett. 52, 1072 (1984).

[20] H. Murayama, H. Suzuki, T. Yanagida, and J. Yokoyama, Phys. Rev. Lett. 70, 1912 (1993).

[21] R. J. Scherrer and M.S. Turner, Phys. Rev. D 33, 1585 (1986).

[22] E. Ma, N. Sahu, and U. Sarkar, J. Phys. G 32, L65 (2006).

[23] M. Malinsky et al. (unpublished). 